JLL 6 (1)(2019)

\title{
LA TRANSFORMATION DU ROMAN UN SAC DE BILLES AU FILM: UNE ETUDE SELON L'ECRANISATION ET LA SEMIOLOGIE
}

\author{
Winarning Pratiwi Saraswati ${ }^{\bowtie}$ Sunahrowi \\ Departement de la Langue et la Littérature étrangere, Faculté des Langues et des Arts, \\ Universitas Negeri Semarang, Indonesia
}

\begin{abstract}
Info d'article Extrait
Histoire de l'article: Reçu fevriér 2019 Accepté avril 2019 Publié mai 2019

Mots-clés:

Roman, Film, Un sac de

billes, Écranisation, Cinq

Codes, Sémiologie

Le roman Un sac de billes est l'un des œuvres de Joseph Joffo. Ce roman prend le thème sur la Seconde Guerre mondiale en France, du point de vue des minorités, les Juifs. L'événement élucidé dans ce roman est La Shoah, l'extermination systématique entre cinq et six millions de Juifs par le Nazi, perpétré sur l'ordre d'Adolf Hitler. Ce roman raconte les deux frères exilés qui ont échappé aux soldats nazis, et ont dû se séparer de leurs familles. Un sac de billes a été publié par les Éditions Jean-Claude Lattès en 1973 et a été traduit en 18 langues jusqu'aujourd'hui. Ce roman est transformé par Christian Duguay au film en 2017. Cette recherche vise à analyser la transformation du roman au film en utilisant la théorie de l'écranisation de Pamusuk Eneste. Cette théorie se compose de la réduction, l'addition, et les changements variés de scène dans le déroulement, le personnage, et le fond. La chercheuse analyse aussi les transformations de l'adaptation Un sac de billes en utilisant la théorie sémiologique de Roland Barthes avec les cinq codes. Ce sont le code herméneutique, le code sémique, le code symbolique, le code proarétique et le code culturel. Les objets matériels dans cette recherche sont le roman Un sac de billes de Joseph Joffo et le film du même titre de Christian Duguay. Tandis que les objets formels sont la théorie de l'écranisation et la théorie sémiologique de cinq codes. La chercheuse utilise 1'approche qualitative avec la méthode d'analyse descriptive. Dans la transformation du déroulement, il y a trente-cinq réductions, vingt-huit additions, et quinze variations. D'ailleurs, dans l'écranisation du personnage, il y a treize réductions, dix additions, et sept variations. Alors, dans l'écranisation du fond, il y a onze réductions, huit additions, et cinq variations. Dans la théorie sémiologique de cinq codes, la chercheuse a découvert trois codes importants, à savoir le code herméneutique, le code sémique et le code proairétique. C'est-a-dire que le réalisateur modifie les scènes du film avec un objectif spécifique: montrer le sens de l'histoire et les connotations cachées dans le roman, et les afficher dans le film dans l'espoir que le spectateur comprenne mieux les signes cachés.
\end{abstract}

(C) 2019 Universitas Negeri Semarang

\begin{tabular}{lr}
\hline Addresse : & ISSN 2252-6730 \\
Gedung B9 Lantai 2 FBS Unnes & \\
Kampus Sekaran, Gunungpati, Semarang, 50229 &
\end{tabular}




\section{L'INTRODUCTION}

La littérature est une œuvre d'art qui est créé par l'auteur ou un groupe de personne en utilisant de la langue. Faruk (2014 : 39) dit qu'étymologiquement, la littérature est une écriture. En anglais, la littérature nommée literature, en allemand assimilée au mot literatur, en français est littérature, et tout cela vient du mot Latin litteratura. Le mot litteratura est une traduction du mot grec grammatika; litteratura et grammatika viennent de mots littera et gramma qui signifie "lettre" (Teeuw, 1984 :20).

Issac et Okunoye (2008: 9) disent que the prose could be fiction or non - fiction. La prose est divisée en deux, à savoir fiction et non-fiction. Selon Aminuddin $(2002: 66)$ la prose fiction est un récit ou l'histoire qui est joué par certaines figures qui comprennent le personnage, le fond, et le déroulement qui viennent d'imagination d'auteur, et deviennent une histoire. L'exemple de la prose fiction est le roman et la nouvelle.

Dans cette recherche, la chercheuse choisira l'un de types de prose fiction c'est le roman. Habituellement, le roman révèle des fragments de la vie humaine qui passe longtemps, où il y a des conflits qui mènent finalement à un changement de vie entre les personnages, principal ou non principal dedans. Muhardi et Hasanuddin WS (1992: 6) montrent qu'un roman est une série de problématiques qui est suivi par la causalité. En autre mot, le roman exprime les problématiques complexes.

La chercheuse va choisir un roman français intitulé «Un sac de billes » de Joseph Joffo et le film «Un sac de billes » de Christian Duguay. Il est basé sur deux raisons : Premièrement, il y a des différences dans le déroulement, le personnage, et le fond entre le roman et le film. Deuxièmement, il y a des raisons cachées dans les transformations de l'adaptation du roman au film.

Basé sur ces raisons au-dessus, il y a deux problèmes principaux dans cette recherche. Ce sont de décrire l'écranisation du déroulement, du personnage, et du fond entre le roman et le film « Un sac de billes ", et alors d'analyser les raisons cachées dans l'adaptation du roman sur l'étude sémiologique de cinq codes de Roland Barthes.

L'écranisation comprend de la réduction, l'addition, et le changement varié. Tandis que les cinq codes de Roland Barthes qui utilise pour analyser les raisons cachées dans l'adaptation du roman sont le code herméneutique, le code sémique, le code symbolique, le code proarétique et le code culturel.

\section{LA THEORIE}

\section{Roman et film}

La langue en tant que le moyen d'œuvre littéraire est ouverte à l'imagination de l'auteur. Beaucoup de processus mentaux se produisent à ce problème. Le langage utilisé permet le lecteur d'interpréter et d'imaginer chacun des événements décrits dans un roman. Alors que dans le film, une durée limitée (entre une heure et deux heures en général) donnera sa propre influence dans le processus de réception et de visualisation des événements du roman. En ce cas, le public se concentrera sur les friandises des cinéastes qui sont réalisées sous la forme d'images, de mouvement et de son. Il est donc moins probable que le public imagine un événement quand il lit un roman.

Selon Sapardi Djoko Damono, l'adaptation cinématographique est un effort pour élargir la portée des amoureux du roman. Eneste (1991: 60) monte que dans le processus de la transformation de l'œuvre littéraire au film, il y a quelques changements. Le roman est une création individuelle et le travail personnel. La prémisse que la littérature traite des cinq sens est, en substance, toute tentative de comparer la littérature avec tout ce qui concerne les cinq sens est une étude utile dans un effort pour comprendre la littérature concernée plus largement et profondément (Damono, 2005: 110). Les 
cinéastes font juste un scénario basé sur l'histoire d'une roman et ensuite versé sous la forme de films bien que la production du film par les cinéastes ne soit pas facile à imaginer.

\section{Écranisation}

La théorie utilisée dans cette recherche est l'écranisation ou l'enlevement d'un roman au film, Eneste (1991: 60). L'écranisation vient de la langue française, à savoir: écran, Eneste (1991: 60). Cette théorie sera utilisée comme un couteau pour montrer le processus de transformation dans un film adapté du roman "Un Sac de Billes". Boogs dans Asrul Sani, (1992: 4-13), a dit que le film reste quelque chose unique, même s'il existe des similitudes avec d'autres médias. Les films dépassent le drame parce que le film a la capacité de prendre une variété de points de vue, allant du mouvement, du temps, et de l'espace illimité. Mangunhardjana (1976: 96) dit que le film peut jouer un rôle en tant que la langue. À travers les images présentées au-dessus de l'écran, le film exprime sa signification, transmet les faits et invite le public à se connecter avec eux.

Loyens (2012:124) dit que le cinéma ne consiste pas seulement à voir, mais à faire voir. Pour cela, non seulement la vision, mais le regarde, la mémoire (il faut se rappeler ce que l'on vient de voir) l'image et ses composantes iconiques, l'imaginaire (ce que j'invente en regardant), le sens, la forme, et le langage participent à la formation au cinéma. C'est à dire que le film apprecié est le film mémorable et inoubliable. Les spectateurs se tiennent les scènes à la mémoire, ils se rappellent les images, le déroulement, les personnages, et les événements dans ses mémoires. La réussite d'un film depend de la compréhension des gens sur l'histoire ou le contenu du film (Pravianti, 2017:14). Ce processus d'écranisation du roman a entraîné de nombreux changements. Eneste (1991: 61-66) explique que les changements, à savoir: la réduction, l'addition et le changement varié.

\section{Réduction}

La réduction est la réduction ou la coupe d'éléments de l'histoire du roman dans le processus de transformation. Eneste (1991: 61) déclare que la réduction peut être faite à des éléments d'œuvres littéraires telles que l'histoire, l'intrigue, le personnage, le contexte et l'atmosphère. Avec le processus de réduction ou de coupe, tout ce qui est révélé dans le roman ne sera pas trouvé dans le film. Ainsi, il y aura des coupures ou des omissions de parties dans l'œuvre littéraire en cours de transformation au film.

\section{Addition}

L'addition est un changement dans le processus de transformation des œuvres littéraires au film. Comme dans la création de la réduction, dans ce processus peut aussi se produire dans l'histoire, le déroulement, les personnages, l'espace et la situation. L'addition fait dans ce processus d'écranisation a certainement une raison. Eneste (1991: 64) montre qu'un réalisateur a une certaine raison d'augmenter dans son film. En outre, certaines additions soutiendront l'aspect commercial qui vise certainement à attirer l'attention du public.

\section{Changement varié}

Le changement varié est la troisième chose qui le rend possible dans le processus de transformation de l'œuvre littéraire au film. Selon Eneste (1991: 65), l'écranisation permet l'apparition de certains changements variés entre le roman et le film. Ici, les changements variés peuvent se produire dans le domaine des idées d'histoire, des styles d'histoire, etc. Le changement varié dans la transformation est influencée par plusieurs facteurs, tels que les médias utilisés, le problème d'audience, la durée du temps. Eneste (1991: 67) montre que les cinéastes doivent produire les changements varies dans le film, donc le film ne ressemble pas beaucoup au roman. 


\section{Sémiologie}

Little John dans Sobur (2006:87), dit que la sémiotique est l'effort de trouver des signes incluant les choses cachées derrière un signe (texte, publicité, nouvelles). La sémiotique est un terme dérivé du mot grec semeion qui signifie «signe» ou sign en anglais, c'est «science qui apprend le système de signe» comme: langage, code, signal, etc. Les signes sont la base de toute communication.

D'après Barthes, la sémiotique est une science ou une méthode d'analyse pour examiner les signes. Les signes sont les outils que nous utilisons pour essayer de trouver un chemin dans ce monde, au milieu des hommes et avec des hommes. La sémiotique, en termes de Barthes, la sémiologie, veut fondamentalement apprendre comment l'humanité (humanity) signifie les choses (things). Signifier (to signify) dans ce cas peut être mélangé avec communiquer (to communicate). Signifier c'est à dire que les objets veulent communiquer, mais constituent aussi un système structuré de signes (Sobur, 2009: 15).

\section{Sémiologie des cinq codes de Roland Barthes}

Selon Barthes, la sémiotique est une science ou une méthode d'analyse pour examiner les signes. Les signes sont les outils que nous utilisons pour essayer de trouver un chemin dans ce monde, au milieu des humains et des humains. (Sobur, 2009: 15) Cette signification n'est pas limitée au langage, mais il y a aussi des choses qui ne sont pas du langage. A la fin, Barthes considérait la vie sociale comme une forme de signification. En d'autres termes, la vie sociale, en aucune forme, est aussi un certain système de signes. La vie sociale est souvent représentée dans le roman et le film. Ainsi, les symboles implicites dans le roman et le film peuvent être transférés par le public dans la vie, et vice versa (Mudjiono, 2011: 130)

Dans son célèbre livre $S / Z$ (1970), Barthes analyse le roman intitulé Sarrasine par Honoré de Balzac. Ce livre est un bon exemple du fonctionnement de Barthes (Sobur, 2009: 65). Barthes tente d'expliciter les codes du récit qui s'appliquent dans un scénario réaliste, il soutient que ce Sarrasine est enfilé dans le code de rationalisation. Les cinq codes revus par Barthes sont:

(1) Le code herméneutique ou le code de puzzle tourne autour de l'espoir du lecteur pour obtenir la «vérité» aux questions qui apparaissent dans le texte.

(2) Le code sémique ou le code connotatif offre de nombreux côtés. Dans le processus de lecture, le lecteur organise le thème d'un texte. Il voit que les connotations de certains mots ou expressions dans le texte peuvent être classifiées avec des connotations de mots ou de phrases similaires.

(3) Les codes symboliques sont l'aspect structurel de l'encodage fictif le plus typique, ou précisément selon Barthes, le concept post structural. Ceci est basé sur l'idée que la signification dérive d'une opposition binaire ou d'une distinction - soit au niveau sonore au phonème dans le processus de production de la parole, soit au niveau de l'opposition psychosexuelle à travers le processus.

(4) Le code proaïrétique ou code d'acte doit être considéré comme l'équipement principal du texte que les gens lisent; c'est-à-dire, tous les textes narratifs. Si Aristoteles et Todorov ne cherchaient que les scènes principales ou le déroulement principal, Barthes voyait théoriquement que tous les codex pouvaient être codifiés, de l'ouverture des portes aux aventures romantiques.

(5) Le code gnomique ou le code culturel est nombreux. Ce code est une référence du texte aux objets déjà connus et codifiés par la culture. Selon Barthes, le réalisme traditionnel est défini par la référence à ce qui est déjà connu.

\section{LA METHODOLOGIE DE LA RECHERCHE}

La méthode et la technique sont des moyens dans un effort. La méthode est le façon qui doit faire dans une analyse, tandis que la technique est le façon d'exécution de la méthode (Sudaryanto dans Muhammad, 2014: 203). Dans cette recherche qualitative, la chercheuse utilisera une méthode d'analyse descriptive. Hidayat (2007: 23) a déclaré que la méthode descriptive est une méthode 
permettant d'examiner le statut d'un groupe de personnes, un objet, une condition, un système de pensée ou un événement. Moleong (2010: 11) a affirmé que les données collectées dans la méthode descriptive sont sous la forme de mots, ou d'images, ce n'est pas sous la forme de nombres. La méthode d'analyse descriptive est réalisée en décrivant des faits qui sont ensuite suivis d'une analyse. Au début, les données sont décrites, pour trouver les éléments, puis les analysées et les comparées (Ratna, 2013: 53). L'analyse des sources de données en utilisant la théorie est la première technique d'analyse des données (Faruk, 2012: 55-56).

En analysant le roman et le film "Un sac de billes ", la chercheuse utilise la théorie de l'écranisation de Pamusuk Eneste. Ensuite, les raisons cachées dans les transformations qui existent dans chaque scène de roman ou de film, il sera analysé plus loin par la chercheuse, en utilisant l'étude sémiologique de Roland Barthes.

\section{L'ANALYSE}

\section{Processus de l'écranisation du roman et du film Un sac de billes}

Dans un film adapté du roman, bien sûr qu'il y a de nombreuses transformations surviennent lors de la visualisation d'un événement du roman dans une scène de film. Ces transformations sont divisées en trois parties principales : les transformations du déroulement, les transformations des personnages et les transformations des fonds. Ensuite, chacun des transformations principales est divisée en trois parties par le processus de l'écranisation, à savoir la réduction, l'addition et le changement varié.

\section{Processus de l'écranisation du déroulement}

Cette partie se divise en trois aspects, ce sont la réduction, l'addition, et le changement varié. Dans la réduction du déroulement il y a trente-cinq données. L'une de ces données est comme cidessous.

La partie suivante se trouve quand l'arrivée des frères Joffo à Dax, accompagnée d'un prêtre qui les a sauvés lors du contrôle de l'armée allemande. Le prêtre les a invités à prendre un petit déjeuner près de la gare de Dax pour se calmer. Joseph et Maurice l'appelaient Monsieur le Curé.

- On va prendre des cafés au lait avec des tartines, dit-il. Mais je vous préviens, le café c'est de l'orge, le sucre c'est de la saccharine, le lait il n'y en a pas, quant aux tartines, il nous faudrait des tickets de pain, mais vous ne devez pas en avoir et moi non plus. Mais cela nous réchauffera tout de même (USdB/147).

La citation au-dessus explique que le prêtre ou Monsieur le Curé les a invités à prendre un café et un petit-déjeuner pour se réchauffer et à calmer les enfants après le contrôle de l'armée allemande. Le réalisateur a choisi de ne pas afficher les petits détails du film.

Alors, il y a vingt-huit données dans l'aspect de l'addition du déroulement. L'une de ces données est trouvée à la scène quand Henri et Albert Joffo lisent une lettre contenant des règles pour les Juifs en France. Le règlement établi par l'armée allemande mentionne la règle selon laquelle les étudiants doivent porter une étoile jaune de David portant l'inscription «Juif» sur leurs vêtements, cousue à la poitrine gauche. 


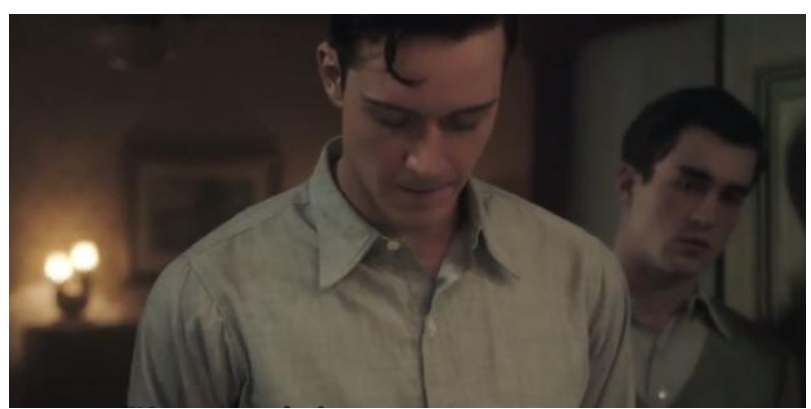

L'addition de la scène ci-dessous est très utile pour que le public puisse ressentir l'émotion en Henri et Albert lors de la lecture de la lettre. Les émotions d'Henri et Albert représentaient également les émotions de la famille Joffo et d'autres familles juives pendant l'occupation allemande de la France.

D'ailleurs, il y a quinze données dans l'aspect du changement varié du déroulement. L'une de ces données est la scène de notification de la Démission de Mussolini. Dans le roman, l'armée milanaise a donné cette information à Joseph peu de temps avant le départ de l'armée italienne de Nice. Nous pouvons voir dans la citation suivante.

Je ne comprenais pas ce qu'il voulait dire. Patiemment, en essayant de faire le minimum de fautes, il m'expliqua :

- Ce n'est plus Mussolini qui commande, c'est Badoglio, et tout le monde se doute qu'il va faire la paix avec les Américains, on dit qu'ils se sont vus déjà, alors si il y a la paix, on rentre chez nous.

L'espoir m'illumina (USdB/477-478).

Il a été expliqué dans la citation au-dessus que l'armée de Milan avait dit à Joseph si Mussolini avait été remplacé par Badoglio, qui avait signé un traité de paix avec l'Amérique. Par conséquent, tous les soldats italiens ont été retirés dans leurs pays.

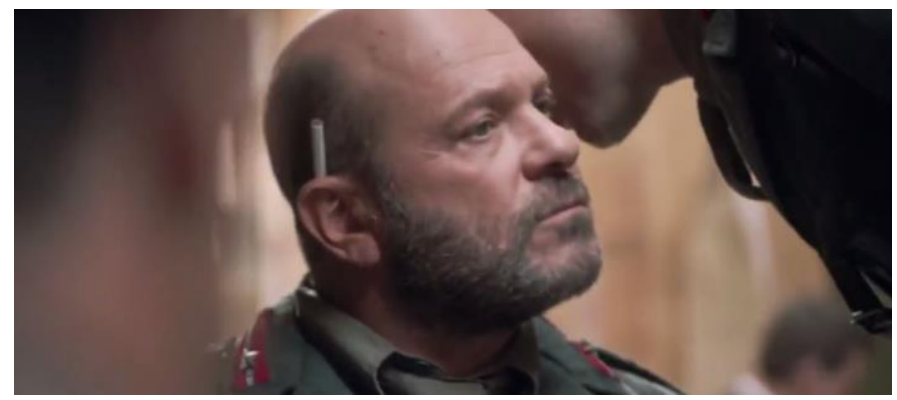

Contrairement aux scènes du film, la notification a été donnée au chuchotement par les amis de Marcello et transmises par Marcello à Monsieur Joffo et à des amis qui jouaient aux cartes. Ce changement entre le roman et le film visent à résumer la durée du film et à économiser le budget de la production.

\section{Processus de l'écranisation du personnage}

Comme l'écranisation du déroulement, cette partie est ausi divisée en trois aspects, la réduction, l'addition, et le changement varié. Dans l'aspect de la réduction du personnage, il y a treize données. L'une de ces données est comme ci-dessous.

$\mathrm{Au}$ debut de l'histoire du roman, il y a le personnage de Mémé Epstein. Mémé Epstein est une femme âgée qui vivait près des écoles de Joseph et de Maurice. Elle est souvent à sa porte en souriant aux écoliers. Il s'est échappé de la Bulgarie, qui vit maintenant dans les rues de Clignancourt. Ceci est montré dans la citation suivante.

Sur le pas de la porte, Mémé Epstein nous regarde. C'est une vieille Bulgare toute ratatinée, ridée comme il n'est pas permis. Elle a bizarrement gardé le teint cuivré que donne au visage le vent des grandes steppes, et là 
dans ce renfoncement de porte, sur sa chaise paillée, elle est un morceau vivant du monde balkanique que le ciel gris de la porte de Clignancourt n'arrive pas à ternir (USdB/12).

Le portrait de Mémé Epstein est apparu lorsque Joseph et Maurice jouaient aux billes avant d'aller à l'école. Mais cette vieille femme sympathique n'est pas montrée dans le film.

En outre, il y a dix données dans l'aspect de l'addition du personnage. L'une de ces données est l'addition de Monsieur Julien. C'était un Savoie, un client du journal et un ami proche de Joseph. Il était présenté comme très en colère contre l'Allemagne et il détestait Monsieur Mancelier, l'hôtesse de Joseph qui était un collabo.

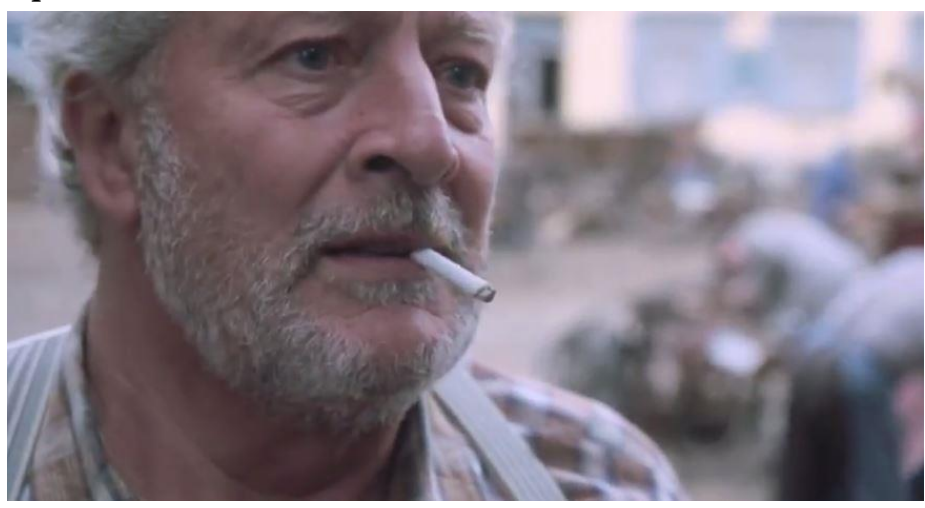

M. Julien n'est pas apparu dans le roman, il a ajouté pour que l'histoire du film était toujours intéressante et que les spectateurs étaient sensibilisés aux sentiments des citoyens sur des collabos, qu'ils n'aimaient pas parce que les collabos se rangeait du côté allemand.

Par ailleurs, dans l'aspect du changement varié du personnage il y a sept données. L'une de ces données est le changement de personnage de Ferdinand. Ce personnage était l'un des employés de Moisson Nouvelle et il travaille souvent en ville pour faire ses courses. C'est décrit dans le roman que Ferdinand était un garçon de vingt-quatre ans et il subissait de tuberculose. Comme on le voit dans la citation ci-dessous.

Ferdinand a vingt-quatre ans, une tuberculose a entraîné quatre ans de sana et l'a fait réformer. Il est l'intendant du centre, le bras droit de Subinagui. Il va régler ces problèmes (USdB/541).

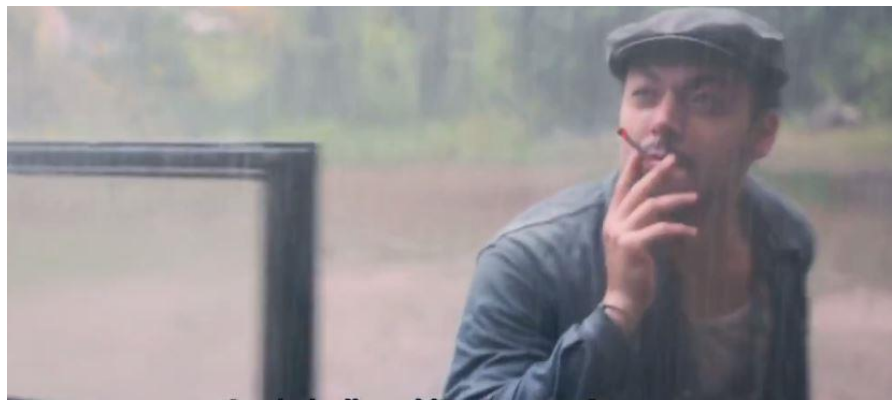

Différent de l'histoire du roman qui décrit Ferdinand comme un homme malade, dans le film, le personnage Ferdinand est décrit comme étant en bonne santé et même s'il fume pendant la pluie, ce que les personnes atteintes de tuberculose ne feront pas. Cela prouve que le personnage de Ferdinand a connu des changements variés d'aspects du comportement.

\section{Processus de l'écranisation du fond}


L'écranisation $d u$ fond est divisée en trois aspects, ce sont la réduction, l'addition, et le changement varié. Il y a onze données dans l'aspect la réduction du fond. L'une de ces données est comme ci-dessous.

C'est la réduction du fond qui est assez grande, à savoir la réduction de la ville Marseille du film. Narré dans le roman, après un long voyage en voiture et à pied, Joseph et Maurice sont arrivés à Marseille. Nous pouvons voir dans la citation suivante.

Bleue, blanche et rose. Il s'en faut de peu que la ville soit de la couleur du drapeau national. Bleu le ciel qui la recouvre, blanches les collines qui l'encerclent et rose pour les toits qui s'étalent, se chevauchent et débutent au bas des escaliers de la gare Saint-Charles. Et au-dessus de tout cela, la tache d'or minuscule de la Vierge de la Garde qui surplombe le tout.

\section{Marseille.}

Je ne me souviens guère du voyage sinon qu'il n'eut rien de comparable avec Paris-Dax. (USdB/253-254)

Marseille n'a pas été montré dans le film Un sac de billes, car une partie de l'histoire se déroulant à Marseille a coupé. Alors bien sûr, cette belle ville est également supprimée de la visualisation du film.

Alors dans l'aspect de l'addition du fond, il y a huit données. L'une de ces données est la scène suivante qui montre la famille Joffo en vacances à la plage. La famille Joffo, qui peut se retrouver à Nice, prend le temps d'aller à la plage. Le fond de la scène est la plage, qui ajoutée pour ajuster l'adition du déroulement.

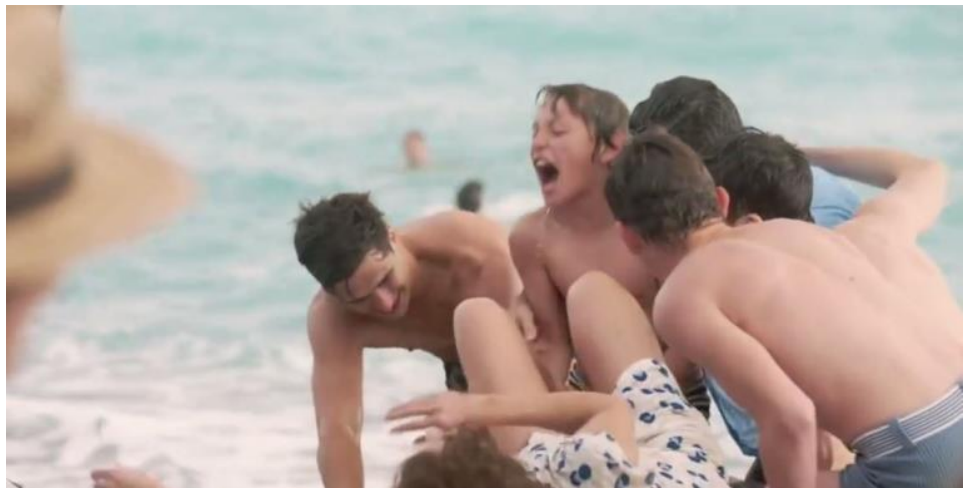

Par ailleurs, il y a cinq données dans l'aspect du changement varié du fond. L'une de ces données est comme ci-dessous.

C'est un changement dans le fond de la scène de l'examen médical à l'hôtel Excelsior. Lors de leur séjour à l'hôtel Excelsior, Joseph et Maurice ont suivi une série de l'examen médical dans une pièce. Comme dans la citation suivante.

Je me retrouve dans une autre pièce, celle-là est vide, il n'y a pas de bureau, il y a trois hommes avec des blouses blanches.

Le plus vieux se retourne lorsque nous entrons (USdB/589).

Dans la citation au-dessus, elle est décrit que la salle de l'examen médical a l'air spacieuse, ne dispose pas de bureau et il n'y a que trois personnes seulement portent des vêtements blancs. Ceci est différent de la description du fond de la scène dans le film. Comme on le voit dans l'image ci-dessous. 


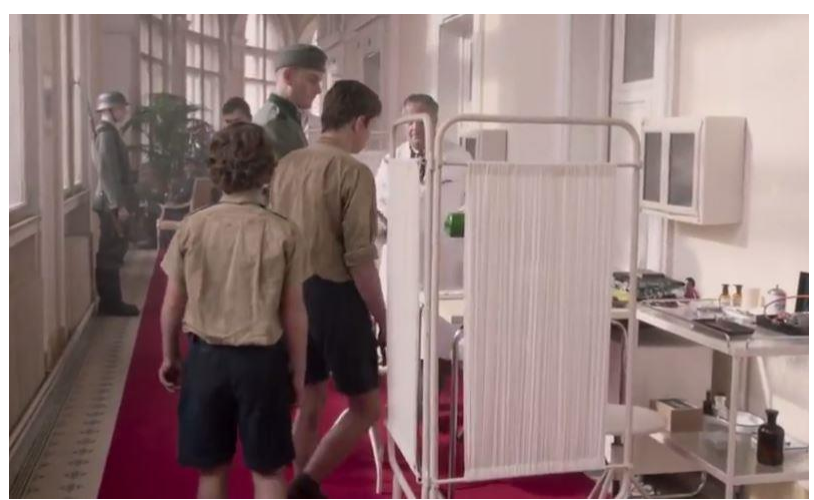

Sur l'image au-dessus, il apparaît que la pièce de l'examen médical est uniquement formée le couloir d'hôtel cloisonné. Le couloir était également encombré et gardé par un soldat. Il s'agit d'un changement varié du fond de la scène fait par le directeur, qui donne l'impression que l'Hôtel Excelsior n'a pas assez place pour la pièce médicale et que l'espace est aménagé avec seulement une petite cloison.

\section{Analyse de 1'adaptation Un sac de billes en utilisant la théorie sémiologique de cinq codes}

Après avoir lu le roman et regardé le film Un sac de billes, nous découvrons le processus de l'écranisation, ainsi que les transformations intervenus dans la scène. En utilisant cinq codes de Barthes, on peut découvrir ce qui se cache derrière le changement d'histoire Un sac de billes. Ensuite on discute profondément des scènes et des parties du roman et du film, et on les regroupera selon les cinq codes de Roland Barthes.

\section{Le titre du roman et du film: $U n$ sac de billes}

L'utilisation du titre Un sac de billes dans le roman et le film est certainement un peu déroutant, car le récit de l'histoire même dans le roman et le film n'a rien à voir avec les billes. Mais apparemment, les billes vont des relations avec Joseph, l'acteur principal.

Le roman raconte que Joseph aime beaucoup jouer aux billes, il a beaucoup de billes et son préféré est les billes d'argile à la surface inégale. Dans le film, ces billes apparaissent à 00:02:42, quand Joseph se souvient de sa fuite en regardant les billes. Alors à 00:03:06, lorsque Joseph les utilisa pour jouer aux billes avec Maurice. Évidemment, ce marbre est spécial parce qu'il est utilisé comme titre de ce roman et de ce film. La raison pour laquelle les marbres ont été choisis comme titre d'une œuvre littéraire est incluse dans le code sémique et le code herméneutique.

Les billes incluent le code sémique car c'est la représentation de la terre pour Joseph. La forme de billes symbolise d'un grand monde, bleu et recoins comme des îles dans la Terre entière.

Et aussi le code herméneutique dans la sélection des billes en tant que titre de ce film est que ces billes sont importantes pour Joseph, l'acteur principal. Un sac des billes correspondent aussi aux mémoires de la vie de Joseph qui deviennent le thème principal du roman et du film. La vie se déroule toujours, comme les billes, en évoquant les souvenir du passé, les souvenir d'enfance de Joseph. Donc le réalisateur maintient ce titre pour le film, car le thème est pareil comme le roman.

\section{Scène de la colère d'Henri avec l'utilisation d'étoile jaune}

Ensuite, la scène d'Henri Joffo qui était en colère quand il a lu la règle que chaque Juif soit obligé d'utiliser une étoile jaune cousue sur la poitrine gauche à l'extérieur de ses vêtements. L'étoile est sous la forme de six points, avec les mots «Juif» au centre. La colère d'Henri a suscité des questions sur le sens véritable de l'utilisation de l'étoile, afin que Christian Duguay puisse ajouter une scène quand Henri était furieux.

L'utilisation d'étoiles hexagonales, également appelée l'étoile jaune, est un signe que les nazis ont donné aux juifs pendant l'occupation par l'Allemagne en France, au cours de la Seconde Guerre 
mondiale. Tous les Juifs à Paris doivent utiliser l'étoile jaune pour faciliter l'identification des autorités. Cette étoile n'est pas simplement une étoile, c'est un signe que celui qui la porte «mérite d'être détruite» aux yeux des nazis. Elle est aussi un symbole de discrimination à l'égard des Juifs en temps de guerre. Dans le film, cette étoile apparaît à 00:09:39 dans la scène où Madame Joffo la coud dans la veste de Joseph, tandis qu'Henri lit les règles d'une voix en colère.

La raison pour l'addition de cette scène est incluse dans le code proairétique, car le réalisateur Duguay a ajouté la scène au-dessus dans le but pour que le spectateur comprenne mieux l'importance d'utilisation de l'étoile pour la famille Joffo. Le choix de Duguay contient également un code herméneutique, car il veut montrer au spectateur le véritable sens de l'étoile jaune et la colère des Juifs tout en l'affichant.

\section{LA CONCLUSION}

D'après l'analyse du processus de l'écranisation sur les éléments du déroulement, du personnage et $\mathrm{du}$ fond dans le roman et le film Un sac de billes, de nombreux changements se produisent, en particulier dans les éléments du déroulement. La plupart des changements se produisent dans la réduction et l'addition du déroulement. La réduction du déroulement est faite car il n'est pas possible d'inclure toutes les parties du roman dans un film. Ensuite, l'addition et le changement varié du déroulement sont faits pour maintenir le déroulement du film ajusté au déroulement du roman. Néanmoins, dans l'ensemble, le déroulement du roman et du film Un sac de billes n'a pas modifié significativement, c'est-à-dire que le déroulement du film a été fait aussi proche que possible avec le déroulement du roman.

D'ailleurs, le processus de l'écranisation sur le personnage et le fond est fait en ajustant les transformations apportées au déroulement. L'addition ou la réduction du personnage et du fond est arrivée qu'il y a certaines parties du roman et du film manquent ou ont été ajoutées. Bien que le changement varié du personnage et du fond soit passé qu'il y a plusieurs raisons qui empêchent de représenter le plus fidèlement possible les personnages et les fonds du roman au film.

Ensuite, après avoir analysé les résultats de l'adaptation du roman "Un sac de billes » en utilisant la théorie sémiologique de cinq codes de Roland Barthes, la chercheuse a découvert trois codes importants, à savoir le code herméneutique, le code sémique et le code proairétique. Le code herméneutique et sémique correspondent aux symboles, signes, et connotations qui cachent, alors le code proairétique correspond aux motifs de chaque acte. C'est-à-dire que le réalisateur modifie les scènes du film avec un motif principal : montrer le sens vérité de l'histoire et les connotations cachées dans le roman, et les afficher dans le film en espérant que le spectateur comprenne mieux les signes cachés.

\section{LA BIBLIOGRAPHIE}

Aziz Alimul, Hidayat. 2007. Metode Penelitian dan Teknik Analisis Data. Jakarta : Salemba Medika.

Aminuddin. 2002. Pengantar Apresiasi Karya Sastra. Bandung : Sinar Baru Algesindo.

Barthes, Roland. 1973. S/Z. Paris : Éditions du Seuil.

Boggs, Joseph M. dan Asrul Sani. 1992. The Art of Watching Film (Cara Menilai Sebuah Film). Jakarta: Yayasan Citra.

Damono, Sapardi Djoko. 2005. Pegangan Penelitian Sastra Bandingan. Jakarta: Pusat Bahasa Departemen Pendidikan Nasional.

Eneste, Pamusuk. 1991. Novel dan Film. Flores: Nusa Indah.

Faruk. 2014. Metode Penelitian Sastra. Yogyakarta: Pustaka Pelajar.. 
Isaac dan Okunoye. 2008. Literature and Literary. Nigeria : University of Nigeria Press. Joffo, Joseph. 1973. Un sac de billes. Paris : Éditions Jean-Claude Lattès. Loyens, Eric . 2012. Du Livre au Film. Point Culture - tdtc.bytenet.it. Mangunhardjana, A. Margiya. 1976. Mengenal Film. Yogyakarta : Yayasan Kanisius. Moleong, Lexy J. 2010. Metodologi Penelitan Kualitatif. Bandung: Remaja Rosdakarya. Mudjiono, Yoyon. 2011. Kajian Semiotika dalam Film. Jurnal Ilmu Komunikasi Vol.1 No.1 Muhardi dan Hasanuddin WS. 1992. Prosedur Analisis Fiksi. Padang: IKIP Padang Press.

Ratna, Nyoman Kutha. 2013. Teori, Metode, dan Teknik Penelitian Sastra. Yogyakarta: Pustaka Pelajar. Sobur, Alex. 2006. Semiotika Komunikasi. Bandung : Remaja Rosdakarya.

Teeuw, Andries. 2003 . Sastra dan Ilmu Sastra. Jakarta : Pustaka Jaya. 